\title{
Vascular and ventilatory mechanical responses in three different stages of pulmonary development in the rabbit model of congenital diaphragmatic hernia ${ }^{1}$
}

Rebeca Lopes Figueira', Karina Miura da Costa", Ana Laura Marsico"', Thamires Melchiades da Silva Milaniv $^{\prime v}$, Walusa Assad Gonçalves ${ }^{v}$, Marcos de Carvalho Borges ${ }^{\mathrm{V}}$, Orlando Castro e Silva ${ }^{\mathrm{VII}}$, Lourenço Sbragia VIII

'Post Doctoral degree, Postgraduate Laboratory of Experimental Fetal Surgery, Division of Pediatric Surgery, Department of Surgery and Anatomy, Ribeirao Preto Medical School, Universidade de São Paulo (USP), Ribeirao Preto-SP, Brazil. Scientific and intellectual content of the study; acquisition, analysis and interpretation of data; technical procedures; histopathological examinations; statistical analysis; manuscript preparation and writing.

"Fellow PhD degree, Postgraduate Laboratory of Experimental Fetal Surgery, Division of Pediatric Surgery, Department of Surgery and Anatomy, Ribeirao Preto Medical School, USP, Ribeirao Preto-SP, Brazil. Technical procedures, manuscript writing, critical revision.

I'Fellow PhD degree, Postgraduate Laboratory of Experimental Fetal Surgery, Division of Pediatric Surgery, Department of Surgery and Anatomy, Ribeirao Preto Medical School, USP, Ribeirao Preto-SP, Brazil. Technical procedures, histopathological examinations.

IVFellow Master degree, Postgraduate Laboratory of Experimental Pulmonary Physiopathology, Department of Internal Medicine, Ribeirao Preto Medical School, USP, Ribeirao Preto-SP, Brazil. Technical procedures, collect and organization of the ventilatory paramethers.

${ }^{\vee}$ Full Professor, Department of Pediatrics, Ribeirao Preto Medical School, USP, Ribeirao Preto-SP, Brazil. Scientific, intellectual and design of the study; technical procedures.

V'Full Professor, Laboratory of Experimental Pulmonary Physiopathology, Department of Internal Medicine, Ribeirao Preto Medical School, USP, Ribeirao Preto-SP, Brazil. Scientific and intellectual content of the study, critical revision, final approval. VIIFull Professor, Laboratory of Liver Transplantation, Department of Surgery and Anatomy, Ribeirao Preto Medical School, USP, Ribeirao Preto-SP, Brazil. Scientific and intellectual content of the study.

VIIIAssociate Professor, Head, Department of Surgery and Anatomy, Laboratory of Experimental Fetal Surgery, Department of Surgery and Anatomy, Ribeirao Preto Medical School, USP, Ribeirao Preto-SP, Brazil. Scientific and intellectual content of the study, manuscript preparation, critical revision, final approval.

\section{Abstract}

Purpose: To evaluate the vascular ventilatory response in different stages of lung development and to compare them to the neonates with congenital diaphragmatic hernia $(\mathrm{CDH})$ in a rabbit model.

Methods: New Zealand rabbits were divided into 8 groups $(n=5)$ : E25, E27, E30, and CDH. All groups were ventilated on a FlexiVent (Scireq, Montreal, QC, Canada), compounding the other 4 groups. The $\mathrm{CDH}$ surgery was performed at E25 and the harvest at E30. Dynamic compliance (CRS), dynamic elastance (ERS) and dynamic resistance (RRS) were measured every $4 \mathrm{~min} / 24$ min. Median wall thickness (MWT) and airspace were measured. ANOVA Bonferroni tests were used to perform statistical analysis. Significance was considered when $p<0.05$.

Results: CRS was higher in E30 compared to all other groups $(p<0.05)$. CRS and RRS of CDH and E27 were similar and were higher in E25 ( $p<0.05)$. MWT was decreased according to the gestational age, was increased in E27V and E30V ( $p<0.05)$ and decreased in CDHV $(p<0.05)$, airspace was decreased in E25 and increased in all ventilated groups $(p<0.05)$.

Conclusions: The ventilation response of congenital diaphragmatic hernia is like the pseudoglandular stage of the lung development. These findings add information about the physiology of pulmonary ventilation in $\mathrm{CDH}$.

Key words: Hernias, Diaphragmatic, Congenital. Lung. Rabbits. 


\section{Introduction}

The pulmonary development in primates has five phases and begins around the fourth week of gestation with the formation of two pulmonary buds derived from the endoderm (embryogenic phase). The primary bronchi and segments are formed by ramifications, which are completed around week 16 (pseudoglandular phase). The lumen of the bronchi and the terminal bronchioles become large, and the vascularization is more prominent between weeks 17-26th (canalicular phase). Between 24 / 26-36th weeks of gestation until the term, an additional subdivision of bronchioles and formation of primitive alveoli occurs (saccular phase). After 36 weeks (alveolar phase), a gas exchange surface increases due to the decrease of the epithelial layer, resulting in thin-walled alveoli ${ }^{1}$. In parallel to the lung development occurs the pulmonary vascular growing, playing an essential role by acting as a limiting factor in branching morphogenesis ${ }^{2}$, also at pseudoglandular pulmonary stage, the fusion of the four components originates the diaphragm: transverse septum (anterior), muscular wall (posterior), pleuroperitoneal membranes (dorsolateral) and esophageal mesentery (dorsal) ${ }^{3}$. When the fusion between the components is not complete, there is a persistence of the pericardial-peritoneal canal, which communicates the chest and the abdomen, resulting in the herniation of the abdomen content to the thoracic cavity defining congenital diaphragmatic hernia $(\mathrm{CDH})^{4}$.

The pulmonary and vascular development are decreased in $\mathrm{CDH}$, resulting in an immature lung and vessels, which characterizes the two main challenges in the disease: the pulmonary hypoplasia and pulmonary hypertension ${ }^{5}$. Pulmonary hypoplasia results from less ramification, both bronchiolar and vascular, causing a decrease in the number of terminal bronchioles and impairment of the development of both lungs, with less severity of the side contralateral to the defect ${ }^{3}$. Pulmonary hypertension $(\mathrm{PH})$ results of a reduction of blood vessels and hyperplasia of the muscular layer of the peripheral vessels associated to an increase in the vasoconstricting response, stimulated by both chemical mediators and by autonomic sympathetic innervation ${ }^{5}$.

In $85 \%$ of cases, $\mathrm{CDH}$ is located in the left posterolateral region (Bochdalek hernia) ${ }^{6}$. The incidence ranges from 0.8-5:10.000 births, with a slight predominance of males, and high mortality rates, requiring multidisciplinary support, extended hospitalization and postdischarge follow-up $\left(^{5,6}\right.$.

The care within the first hours of life is critical for newborns with $\mathrm{CDH}$. It may involve strategies such as immediate intubation, nasogastric tube, hemodynamic stabilization, sedation, anesthesia and mechanical ventilation, associated or not with inhaled nitric oxide (NO) and extracorporeal membrane oxygenation (ECMO) [9]. Due to the respiratory particularities of the newborn with $\mathrm{CDH}$, mechanical ventilation is considered a challenge, with damages associated with its prolonged use, oxygen toxicity, bronchopulmonary dysplasia and excessive pulmonary distension, which are called ventilation-induced lung injury ${ }^{10}$. According to experimental studies, its pathophysiology associates with residual volumes and larger PEEP, i.e., excessive enlargement of the alveoli and their repetitive reopening ${ }^{11}$.

Experimental models of $\mathrm{CDH}$ are essential to study the etiology, physiopathology, and to develop new strategies of treatment in this field of research ${ }^{12}$. The most commonly used animal models for $\mathrm{CDH}$ are rats, mouses, sheep and rabbits $^{13}$. In rats and mice, the primary models are the toxicological or genetic knockout, contributing to the investigation of CDH's etiology, especially in pathways of the diaphragm development ${ }^{3,14,13}$. The surgical model is usually performed in bigger animals 
to study the mechanical effect of the viscera on the airways, as hypoplasia and pulmonary hypertension. In rabbits, the creation of the defect is on day 25 (term = 30 days), at the end of pseudoglandular and the beginning of canalicular phases on the rabbit pulmonary development stage, corresponding to the 20th week of gestation in humans (canalicular phase) ${ }^{14}$.

Considering the importance of the pulmonary vasculature response in the study of $\mathrm{CDH}$ and its alterations after ventilation, this study aimed to verify the parameters of respiratory dynamics and the median wall thickness changes in the $\mathrm{CDH}$ /ventilatory model in rabbits in three different stages of pulmonary development.

\section{- Methods}

The study was approved by the ethics committee of studies in animal experimentation (CEUA) of Ribeirão Preto Medical School, protocol \# 100/2017.

The animals were kept at the experimental surgery vivarium at the appropriate temperature and light for two weeks and received ration for rabbits, and water ad libitum until the harvest.

\section{Experimental groups}

New Zealand rabbits were divided into eight experimental groups $(n=5$ rabbits per group):

E25: control harvested at embryonic day 25th;

E25V: ventilated control harvested at embryonic day 25th;

E27: control harvested at embryonic day 27th;

E27V: ventilated control harvested at embryonic day 27th;

E30: control harvested at embryonic day 30th (term);

E30V: ventilated control harvested at embryonic day 30th (term);

$\mathrm{CDH}$ : fetus submitted to $\mathrm{CDH}$ induction on embryonic day 25th, harvested on embryonic day 30th $(n=5)$;

CDHV: neonates submitted to $\mathrm{CDH}$ induction on embryonic 25th, submitted to mechanical ventilation after the cesarian section on embryonic 30th (Figure 1).

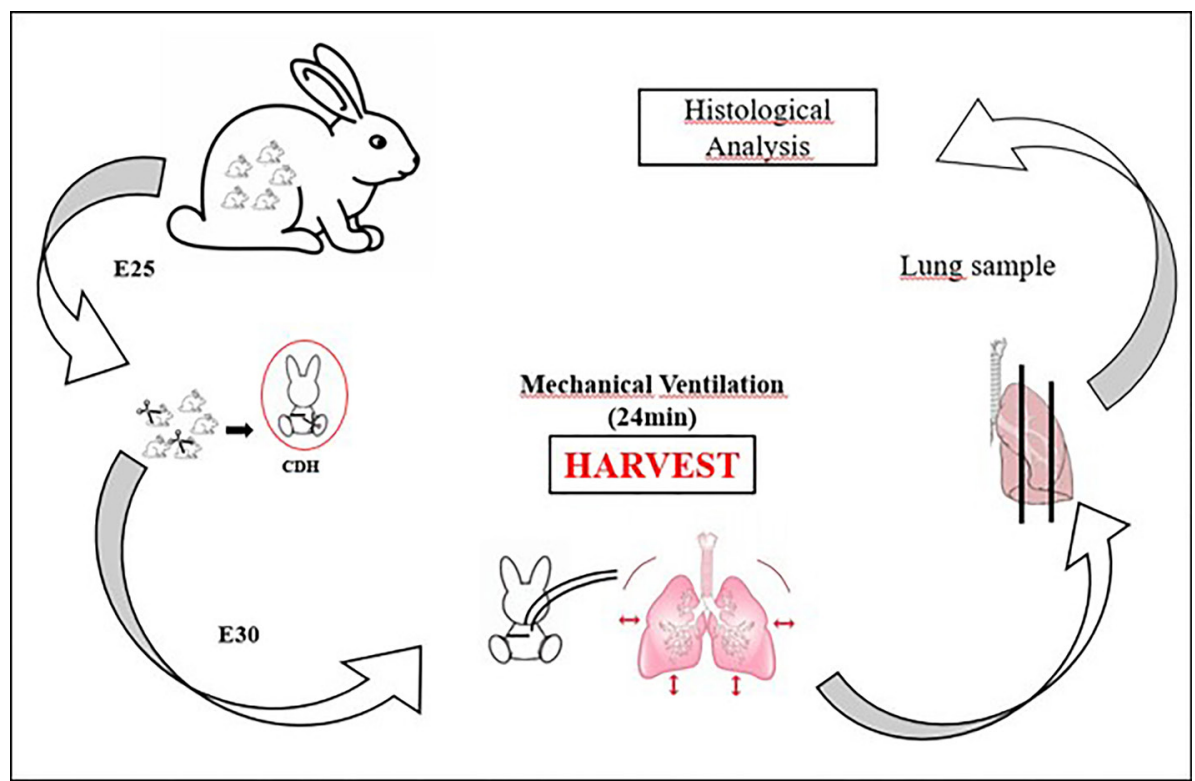

Figure 1 - Study design of CDHV group. E25: embryonic day $25^{\text {th }}$. CDH: congenital diaphragmatic hernia. E30: embryonic day $30^{\text {th }}$. 


\section{Surgical procedure}

All New Zealand rabbits were duly weighed and cataloged in their chart. The surgical procedure to create the $\mathrm{CDH}$ occurred on day 25 (E25) (term = 30 days), with the pregnant rabbits weighing between 3.7 and $4.5 \mathrm{~kg}$. Induction and anesthetic maintenance were performed by intramuscular injection of ketamine sulfate (ketamin ${ }^{\circ}$ ) 25 to $50 \mathrm{mg} /$ $\mathrm{kg}$ associated with xylazine hydrochloride (Rompun-Bayer) 5 to $10 \mathrm{mg} / \mathrm{kg}$ in the right posterior thigh. This dose allowed proper anesthesia for 30 to 40 minutes. If necessary, an additional dose was given when the surgical procedure exceeded this period, with half the initial volume. The pregnant rabbit was placed on a surgical thermal table at a constant temperature of $38^{\circ} \mathrm{C}$ (Harvard Apparatus ${ }^{\circ}$ ), with $100 \%$ oxygen (mask) 1-2 L/min which remained throughout the surgical procedure until total anesthetic recovery. After that, the abdominal region was prepared with an alcoholic solution of chlorhexidine $2 \%$ (Riohex $2 \%{ }^{\circ}$ ) and was operated under aseptic conditions by two surgeons. Before incision, four $\mathrm{ml}$ of Lidocaine $1 \%$ without vasoconstrictor (Xylestesin ${ }^{\circ}$ ) was injected in the hypodermic and muscular planes. A median infra-umbilical laparotomy was performed. The uterus was identified (rabbits have uterus bicorne), and one of the horns was gently exposed outside the abdominal cavity. The fetuses were identified from the ovary towards the cervix. The fetus closest to the ovary was denominated fetus 1 , and the next fetus (fetus 2) was the control fetus. The surgical procedure was performed in each uterine horn, in a total of 2-4 fetuses with HDC per rabbit.

\section{Surgical creation of diaphragmatic hernia}

The procedure was performed
according to Fauza et al. ${ }^{16}$, on E25. After

identification of the fetal position, a circumferential suture was performed with Prolene $5-0^{\circ}$ at the level of the fetal thorax, which was seen by transparency through the uterus. After opening the uterine wall, the paw of the left anterior limb was gently exposed. A small dose $(0.2 \mathrm{ml})$ of Lidocaine $1 \%$ without vasoconstrictor (Xylestesin ${ }^{\circ}$ ) was injected into the hypodermic/muscular planes in the posterolateral region of the left thorax, followed by a $3 \mathrm{~mm}$ thoracotomy, with exposure of the diaphragm which was incised. The chest was closed with Prolene $6-0$, and 2 ml of warm saline $(\mathrm{NaCl} 0.9 \%)$ was administered into the amniotic cavity before closure of the uterine wall to replenish the lost amniotic fluid (Figure 2). Throughout the surgical procedure, the uterine horn was irrigated with a warm saline solution ( $\mathrm{NaCl} 0.9 \%)$. After completion of the fetal $\mathrm{CDH}$, the uterine horn was re-inserted into the abdominal cavity, and the same procedure was performed on other uterus horn. Finally, the uterine cavity was closed with $\mathrm{Vicryl}^{\circ}$ 2-0, and the skin with Nylon ${ }^{\circ}$ 4-0 (continuous intradermal suture). At the end of the procedure, the animals received $25 \mathrm{mg} / \mathrm{kg}$ Cefazolin and $2.5 \mathrm{mg} / \mathrm{kg}$ Medroxyprogesterone Acetate (Depo Provera ${ }^{\circ}$ ) intramuscularly. After recovery, the pregnant rabbit was returned to the vivarium, where it remained for another five days until the final harvest, which occurred on E30. All the anesthetic procedure was the same as the maternal surgery, and the doses were adjusted to the offspring according to the fetal and neonatal weight. After the abdominal and uterine opening of the rabbit, the fetuses submitted to $\mathrm{CDH}$ and controls were removed from the uterus, cleaned and weighed on an analytical balance (model Ohaus APX-200). After harvesting the required material, the anesthetized rabbit was sacrificed with a lethal dose of Thionembutal in the auricular vein. 


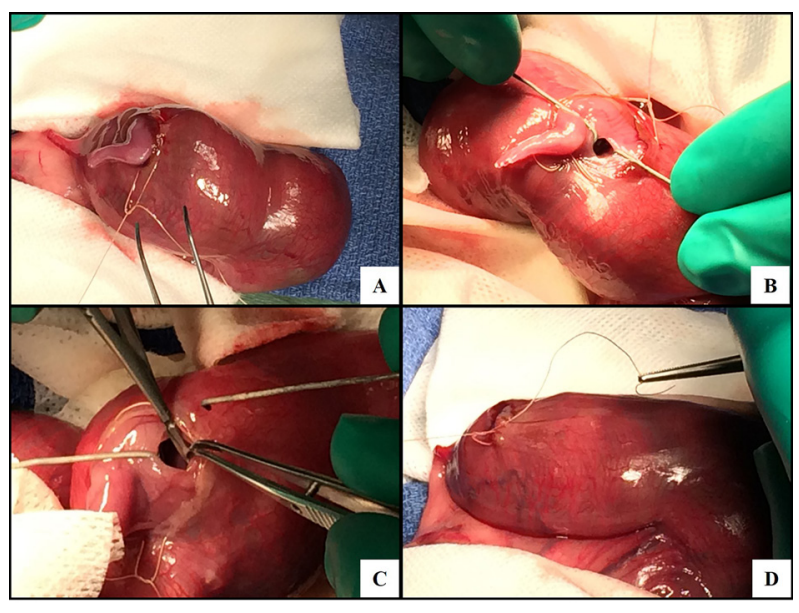

Figure 2 - Surgically creation of $\mathrm{CDH}$. A) Opened uterine wall with exposure of the left anterior limb of the fetus. B) Left posterolateral thoracotomy. C) Section of the diaphragm. D) Closure of the uterine wall.

\section{Mechanical ventilation and in vivo lung ventilatory parameters}

The rabbits were anesthetized on the specific gestational day to complete the groups (E25V, E27V, E30V or CDHV) by intramuscular injection of ketamine (ketamin $\left.{ }^{\circ}\right) 25$ to 50 $\mathrm{mg} / \mathrm{kg}$ associated with xylazine (Rompun ${ }^{\circ}$ Bayer) 5 to $10 \mathrm{mg} / \mathrm{kg}$ intramuscularly in the right posterior thigh. A median laparotomy was performed in the same location as the previous surgery. After identification of the fetus with $\mathrm{CDH}$, fetal removal was conducted through a uterine incision. The neonate was weighed and received $1 \mathrm{ml}$ of intraperitoneal ketamine. It was placed in a supine position on a heated table. After cervicotomy, the trachea was isolated, and tracheostomy was performed with a vascular $18 \mathrm{G}$ catheter (BD), which was connected to FlexiVent (Scireq, Montreal, QC, Canada). The parameters used for ventilation were: the respiratory rate of 150 breaths/minute, PEEP (positive end-expiratory pressure) of $4 \mathrm{~cm} \mathrm{H} \mathrm{H}_{2} \mathrm{O}$, inspiratory time of $0.1 \mathrm{~s}$ and expiratory time of $0.3 \mathrm{~s}$. Pulmonary mechanics were measured every 4 min for 16 $\min$, and, whenever possible, for $24 \mathrm{~min}^{17}$. The animals had their temperature checked every 10 minutes. For this purpose, they were kept on a thermal mattress (adult rabbits) / table (newborn rabbits) and the neonates were wrapped in plastic for thermal maintenance (Figure 3).

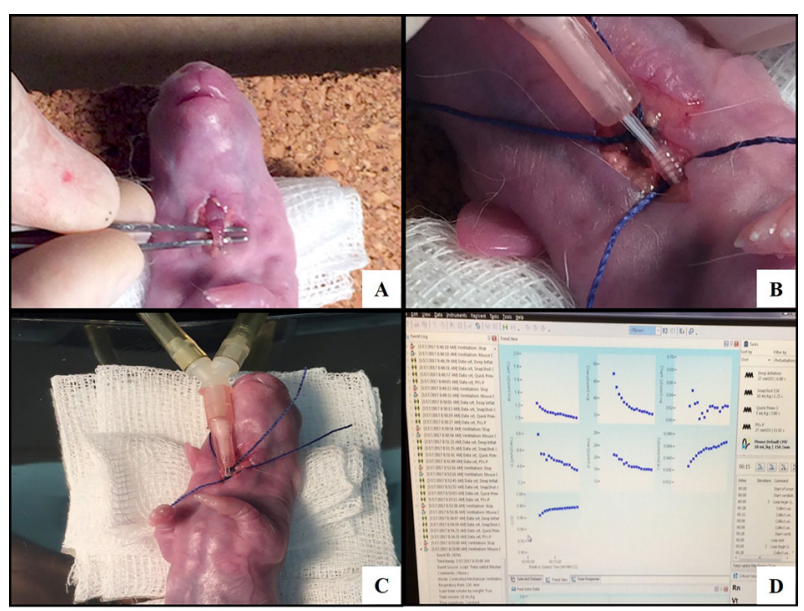

Figure 3 - Mechanical ventilation. A) Cervicotomy and tracheal identification. B) Tracheostomy with the vascular catheter. C) Connection to the FlexiVent. D) FlexiVent software data.

\section{Harvest and sample processing}

After ventilation, the neonates were anesthetized by intramuscular injection of ketamine (ketamin $\left.{ }^{\circ}\right) 25$ to $50 \mathrm{mg} / \mathrm{kg}$ plus xylazine (Rompun -Bayer) 5 to $10 \mathrm{mg} / \mathrm{kg}$ intramuscularly in the right posterior thigh. The lung harvest was performing according to the American Society of Thoracic Studies, which suggests the lung collect after the pressure of 20-25 $\mathrm{mmHg}$ with formaldehyde through the airway, followed by immediate placement in formaldehyde.

Fixation was in formaldehyde 10\%; the samples were dehydrated in increasing ethanol gradient $(70 \%, 80 \%, 90 \%$ and $100 \%$, respectively), diaphanized in xylol and included in histological paraffin. The histological 
sections were performed on a Leica microtome (Model RM 2145), with $5 \mu \mathrm{m}$ thickness. They were stained with Masson Trichrome (MT) and mounted on Entellan'.

\section{Lung and vascular morphometry}

For this study, we used left lung samples. The lung sections stained with MT were photographed on a Nikon photomicroscope (Eclipse 80i - Nikon Instruments Inc. Melville, NY, USA) with magnification of 200x and the images were analyzed using Image J2 (National Institutes of Health, Bethesda, MD, USA), a total of 60 sections of lung per group were analyzed. First, image scaling was defined with the Analyze-Set Scale command. The images were analyzed using the color threshold function using Image - Adjust - Color Threshold - Analyze - Measure. Airspace value was measured by subtracting the total parenchyma area minus the total area. Two fields were analyzed per neonate ( $n=6$ per group). The results were expressed in pixel/ $\mu \mathrm{m}$.

For the pulmonary vasculature analyze, was used a total of 40 arterial histological sections per group were photographed with a magnification of 400X and analyzed using Image-Pro Plus 6.0 software (Media Cybernetics Inc., Rockville, MD-USA). Preacinar arterioles between 0 and $30 \mu \mathrm{m}$ were included. The external diameter (ED) and the internal diameter (ID) were measured, and the media wall thickness (MWT) was calculated using the formula: MWT = (ED-ID) $/ E D^{18}$.

\section{Statistical analysis}

Statistical comparisons were made using one-way analysis of variance (ANOVA), and the Bonferroni method was established as a post hoc test. Student's t-test was also used when appropriate. Significance values were considered when $P<0.05$. The program used for statistical analysis was GraphPad Instat, 1997, version 3.0 (GraphPad Prism Software, San Diego, CA, USA).

\section{- Results}

\section{Mechanical ventilation and in vivo lung ventilatory parameters}

The ventilatory parameters obtained were: dynamic compliance (CRS), dynamic elastance (ERS) and dynamic resistance (RRS). Results showed increased CRS, from the higher to the lower, E30V, CDHV, E27V and E25V, respectively $(P<0.05)$. RRS was increased in E25V group compared to all other groups $(P<0.05)$, and in the group E30V RRS was lower compared to all other groups $(P<0.05)$, with no difference between the groups E27V and CDHV (NS). ERS was increased in the group E25V compared to all groups $(P<0.05)$, and decreased in E27V, E30V, and CDHV with no difference in the after $15 \mathrm{~min}$ of ventilation. Figure 4 shows the graphs of the respective analyzes.
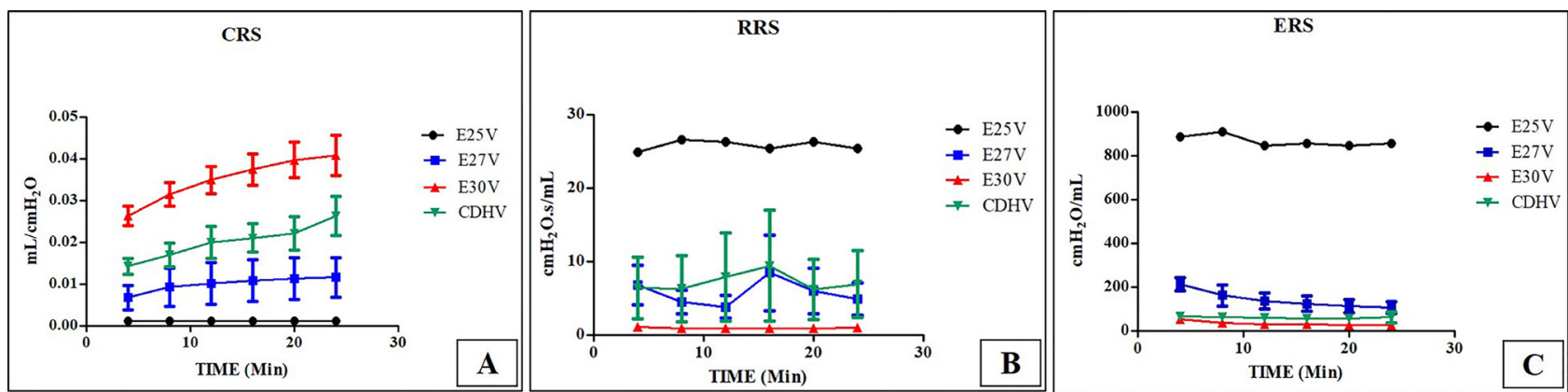

Figure 4 - Ventilatory parameters analyzed. A) CRS: dynamic compliance. B) RRS: dynamic resistance. C) ERS: dynamic elastance. ${ }^{*} p<0.05 .{ }^{* *} p<0.005$. CDHV: group $\mathrm{CDH}$ submitted to mechanical ventilation. 


\section{Lung and vascular morphometry}

The morphometric analysis of the lung parenchyma showed increased area of airspace in the groups ventilated compared to the nonventilated ones, E27 vs. E27V $(P<0.005)$, E30 vs. E30V $(\mathrm{P}<0.05), \mathrm{CDH}$ vs. CDHV $(\mathrm{P}<0.05)$, except for the E25 vs. E25V groups (NS) that did not present difference (Figures 5 and 6 ). The group $\mathrm{CDH}$ and group $\mathrm{E} 30$ were not different in airspace (NS).

MWT decreased gradually according to the gestational age (E25, E27, E30) and it was influenced by mechanical ventilation, except in the E25V group that did not change compared to E25 group (NS). E27V presented increased MTW compared to E27 $(P<0.005)$, E30V presented increased MTW compared to E30 ( $\mathrm{P}<0.005)$, and CDHV presented decreased MWT compared to $\mathrm{CDH}(\mathrm{P}<0.005)$ (Figure 7).

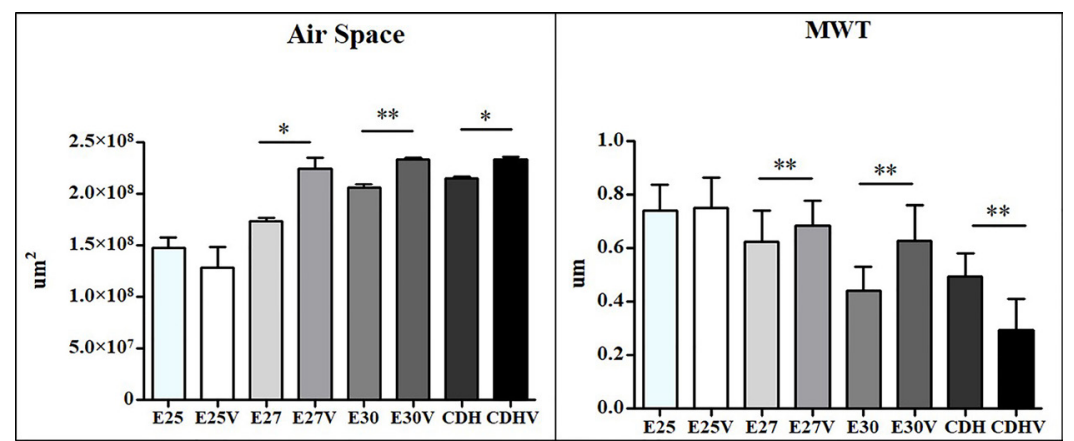

Figure 5 - Airspace and MWT analyzes. A) Graphic of Air Space Area. Unit: $\mu \mathrm{m}^{2}$. B) Graphic of MWT. Unit: $\mu \mathrm{m}$. $* P<0.05, * * P<0.005$.

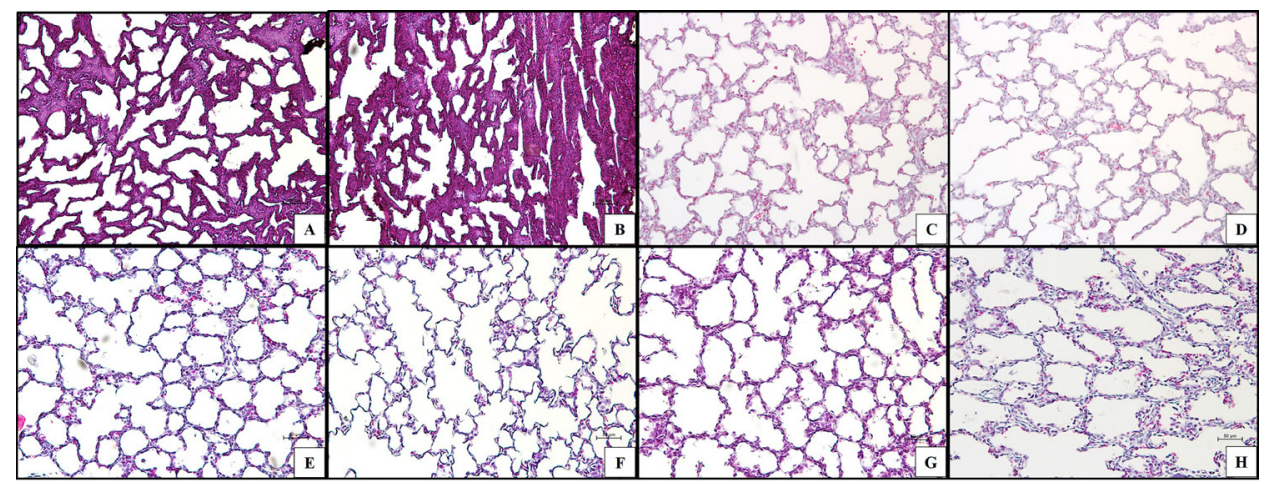

Figure 6 - Masson Trichrome staining of lung tissue sections. A) Group E25. B) Group E25V. C) Group E27. D) Group E27V. E) Group E30. F) Group E30V. G) Group CDH. H) Group CDHV Magnification: x20. Bar: 50 $\mu$ m.

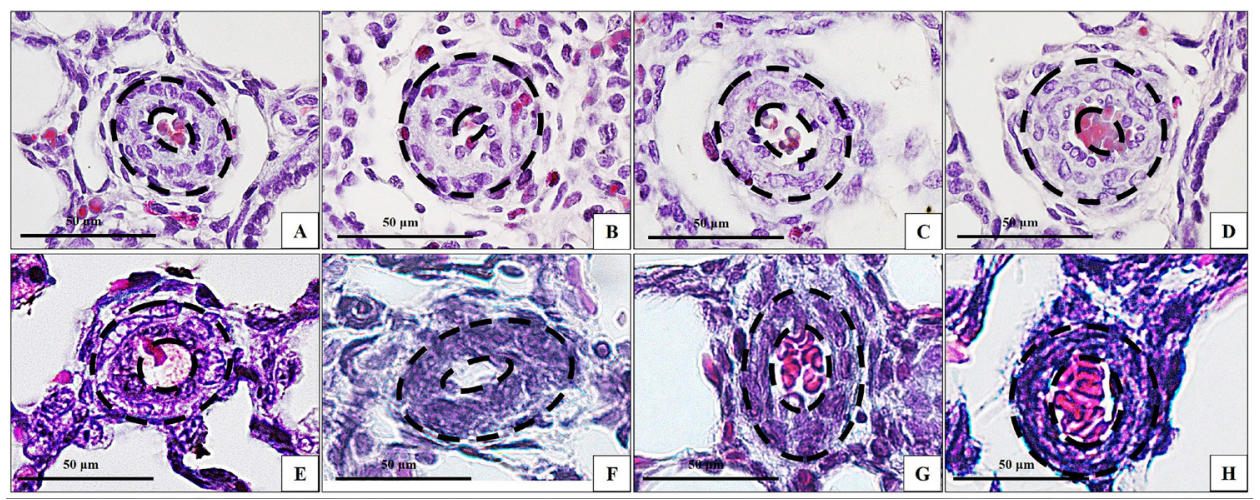

Figure 7 - Pulmonary vasculature. A) Group E25. B) Group E25V. C) Group E27. D) Group E27V. E) Group E30. F) Group E30V. G) Group CDH. H) Group CDHV. Black lines point the median wall thickness. Magnification: x40. Bar: $50 \mu \mathrm{m}$. 


\section{- Discussion}

In this study, we verified the pulmonary arteriolar response in three different stages of lung development (pseudoglandular, canalicular and alveolar) and compared the results with the ventilatory parameters in the rabbit model of $\mathrm{CDH}$.

The ventilatory model in rabbits with $\mathrm{CDH}$ it is not new. However, there are few studies in the literature on the vascular response in different stages of pulmonary development in this model. Ohi et al. ${ }^{19}$ compared the pulmonary growth in the 30th day of gestation (term) with the immature lung in the 25th day of pregnancy, the comparison of Ohi et al. ${ }^{19}$ was focused on hypoplasia signals on parenchyma evaluation, the conclusion proved the point of a smaller lung, however, with no definite signs of hypoplasia, indicating a lung with probable normal function. We did not evaluate the pulmonary hypoplasia in our study; we measured the airspace as an indirect morphometrical analysis of pulmonary immaturity. Our results showed no difference between the groups $\mathrm{CDH}$ and $\mathrm{E30}$, which may be explained by the pressure fixation protocol of the lungs, a technical issue in the rabbit model of $\mathrm{CDH}$ already published by Roubliova et $a{ }^{20}{ }^{20}$ that compared the littermates tissue with the non-littermates tissues, showing peculiar differences as in the LMW (wall thickness) measurements.

Currently, Flemmer et al. ${ }^{13}$ studied the neonatal pulmonary impedance in the rabbit model of CDH related to the severity of lung hypoplasia using a ventilatory parameter called forced oscillation technique (FOT), which determine lung tissue forces in small animals, the results showed FOT as good parameter indicator of hypoplastic lungs. In our study, we may say that the ventilatory parameters pointed CDH with similar responses (NS) of E27 (pseudoglandular phase), especially by reveling equal response in two parameters (RRS and ERS) and decreased CRS. An ERS is numerically equivalent to the inverse of the CRS, being consistent with the values obtained from CRS. RRS, in turn, corresponding to the ratio of resistive pressure by the respiratory system to air in the alveoli and air ${ }^{13,21}$.

However, the arteriolar response after ventilation was different between these two groups (CDH and E27), with MWT decreased in the group $\mathrm{CDH}$ and increased in the group E27. MWT response after ventilation pointed the acute use of oxygen as a good vasodilator for $\mathrm{CDH}$, but not for the other groups, which presented vasoconstriction after $24 \mathrm{~min}$ of ventilation. In the groups non-ventilated the $\mathrm{CDH}$ group presented higher WMT than E30 (term control) as showed in the model of $\mathrm{CDH}$ in rats, the $\mathrm{CDH}$ vascular responses after ventilation presented vasodilatation, while Control group presented vasoconstriction ${ }^{15}$. Other studies in literature also show the increased arteriolar thickness in the rabbit and the rat model of $\mathrm{CDH}$, as Roubliova et $a .^{22}$ and Taira et al. ${ }^{23}$, respectively.

In this study it was possible to identify that mechanical ventilation modulates the response of the parenchyma and the pulmonary arteriolar vasculature. Also the ventilation response of $\mathrm{CDH}$ is similar to the pseudoglandular stage of the lung development (E27), concerning compliance, elastance, and resistance. The median wall thickness response on the immature lungs, E25 and E27, were similar to the E30 after ventilation, showing, when not submitted to ventilation, a gradual decrease of the muscle layer according to the gestational age.

\section{Conclusion}

These findings add information about the pulmonary mechanical influence and alterations after pulmonary ventilation in $\mathrm{CDH}$ 
and accomplish new information about the $\mathrm{CDH}$ model in rabbits.

\section{References}

1 Mayer S, Metzger R, Kluth D. The embryology of the diaphragm. Semin Pediatr Surg. 2011;20:161-9. doi: 10.1053/j. sempedsurg.2011.03.006.

2 Mous DS, Kool HM, Wijnen R, Tibboel $D$, Rottier RJ. Pulmonary vascular development in congenital diaphragmatic hernia. Eur Respir Rev. 2018;26:147. doi: 10.1183/16000617.0104-2017.

3 Gallindo RM, Gonçalves FL, Figueira RL, Sbragia L. Prenatal management of congenital diaphragmatic hernia: present, past and future. Rev Bras Ginecol Obstet. 2015;37:140-7. doi: 10.1590/S0100720320150005203.

4 van Loenhout RB, Tibboel D, Post $M$, Keijzer R. Congenital diaphragmatic hernia: comparison of animal models and relevance to the human situation. Neonatology. 2009;96:137-49. doi: 10.1159/000209850.

5 Yamataka T, Puri P. Pulmonary artery structural changes in pulmonary hypertension complicating congenital diaphragmatic hernia. J Pediatr Surg. 1997;32:387-90. doi: 10.1016/S00223468(97)90587-X.

6 Kotecha S, Barbato A, Bush A, Claus F, Davenport $M$, Delacourt C, Deprest J, Eber E, Frenckner B, Greenough A, Nicholson AG, Antón-Pacheco JL, Midulla F. Congenital diaphragmatic hernia. Eur Respir J. 2012;39:820-9. doi: 10.1183/09031936.00066511.

7 Chandrasekharan PK, Rawat M, Madappa $\mathrm{R}$, Rothstein $\mathrm{DH}$, Lakshminrusimha $\mathrm{S}$. Congenital diaphragmatic hernia - a review. Matern Heal Neonatol Perinatol. 2017;3:6. doi: 10.1186/s40748-017-0045-1.

8 Varisco BM, Sbragia L, Chen J, Scorletti F, Joshi R, Wong HR, Lopes-Figueira R, Oria $M$, Peiro J. Excessive reversal of epidermal growth factor receptor and ephrin signaling following tracheal occlusion in rabbit model of congenital diaphragmatic hernia. Mol Med. 2016;22:1. doi: 10.2119/ molmed.2016.00121.

9 Snoek KG, Capolupo I, van Rosmalen J,
Hout L de J den, Vijfhuize S, Greenough A, Wijnen RM, Tibboel D, Reiss IK. Conventional mechanical ventilation versus high-frequency oscillatory ventilation for congenital diaphragmatic hernia. Ann Surg. 2016;263:867-74. doi: 10.1097/ SLA.0000000000001533.

10 Logan JW, Cotten CM, Goldberg RN, Clark $\mathrm{RH}$. Mechanical ventilation strategies in the management of congenital diaphragmatic hernia. Semin Pediatr Surg. 2007;16:115-25. doi: 10.1053/j.sempedsurg.2007.01.006.

11 Imai Y, Nakagawa S, Ito Y, Kawano T, Slutsky AS, Miyasaka K. Comparison of lung protection strategies using conventional and high-frequency oscillatory ventilation. J Appl Physiol. 2001;91:1836-44. doi: 10.1152/jappl.2001.91.4.1836.

12 Beurskens N, Klaassens $M$, Rottier $R$, de Klein A, Tibboel D. Linking animal models to human congenital diaphragmatic hernia. Birth Defects Res Part A Clin Mol Teratol. 2007;79:565-72. doi: 10.1002/bdra.20370.

13 Flemmer AW, Jani JC, Bergmann F, Muensterer OJ, Gallot D, Hajek K, Sugawara J, Till H, Deprest JA. Lung tissue mechanics predict lung hypoplasia in a rabbit model for congenital diaphragmatic hernia. Pediatr Pulmonol. 2007;42:505-12. doi: 10.1002/ ppul.20618.

14 Pringle KC. Human fetal lung development and related animal models. Clin Obstet Gynecol. 1986;29:502-13. PMID: 3757332.

15 Gallindo RM, Gonçalves FLL, Figueira RL, Pereira LAVD, Simões ALB, Schmidt AF, Sbragia L. Ventilation causes pulmonary vascular dilation and modulates the NOS and VEGF pathway on newborn rats with CDH. J Pediatr Surg. 2015;50:842-8. doi: 10.1016/j.jpedsurg.2014.12.005.

16 Fauza DO, Tannuri U, Ayoub AA, Capelozzi VL, Saldiva PH, Maksoud JG. Surgically produced congenital diaphragmatic hernia in fetal rabbits. J Pediatr Surg. 1994;29:8826. PMID: 7931963.

17 Debeer A, Sbragia L, Vrancken K, Hendriks A, Roubliova X, Jani J, Naulaers G, Carmeliet P, Deprest J. Antenatal fetal VEGF therapy to promote pulmonary maturation in a preterm rabbit model. Early Hum Dev. 2010;86:99-105. doi: 10.1016/j. earlhumdev.2010.01.025.

18 Dingeldein M. Congenital diaphragmatic 
hernia. Adv Pediatr. 2018;65:241-7. doi: 10.1016/j.yapd.2018.05.001.

19 Ohi R, Suzuki H, Kato T, Kasai M. Development of the lung in fetal rabbits with experimental diaphragmatic hernia. J Pediatr Surg. 1976;11:955-9. doi: 10.1016/ S0022-3468(76)80073-5.

20 Roubliova XI, Biard JM, Ophalvens L, Gallot D, Jani JC, Verbeken EK, van de Ven CP, Tibboel $D$, Deprest JA. Morphology of the developing fetal lung the rabbit experimental model. Mod Res and Educ Top in Micr. 2007.

21 Morini F, Capolupo I, van Weteringen W, Reiss I. Ventilation modalities in infants with congenital diaphragmatic hernia. Semin Pediatr Surg. 2017;26:159-65. doi: 10.1053/j.sempedsurg.2017.04.003.

22 Roubliova XI, Deprest JA, Biard JM, Ophalvens L, Gallot D, Jani JC, van de Ven CP, Tibboel $D$, Verbeken EK. Morphologic changes and methodological issues in the rabbit experimental model for diaphragmatic hernia. Histol Histopathol. 2010;25:110516. doi: 10.14670/HH-25.1105.

23 Taira Y, Miyazaki E, Ohshiro K, Yamataka $\mathrm{T}$, Puri P. Administration of antenatal glucocorticoids prevents pulmonary artery structural changes in nitrofen-induced congenital diaphragmatic hernia in rats. J Pediatr Surg. 1998;33:1052-6. doi: 10.1016/ S0022-3468(98)90530-9.

\section{Correspondence:}

Prof. Dr. Lourenço Sbragia

Disciplina de Cirurgia Pediatrica

Departamento de Cirurgia e Anatomia, USP

Avenida Bandeirantes, 3900

14049-900 Ribeirão Preto - SP Brasil

Tel.: (55 16)3602-2593 / 3602-2501

sbragia@fmrp.usp.br

Received: June 09, 2018

Review: Aug 12, 2018

Accepted: Sept 08, 2018
Conflict of interest: none

Financial sources: FAPESP, CNPq, and CAPES

\footnotetext{
${ }^{1}$ Research performed at Laboratory of Fetal and Neonatal Experimental Surgery, Division of Pediatric Surgery, Department of Surgery and Anatomy, Ribeirao Preto Medical School, Universidade de São Paulo (USP), Brazil.
} 